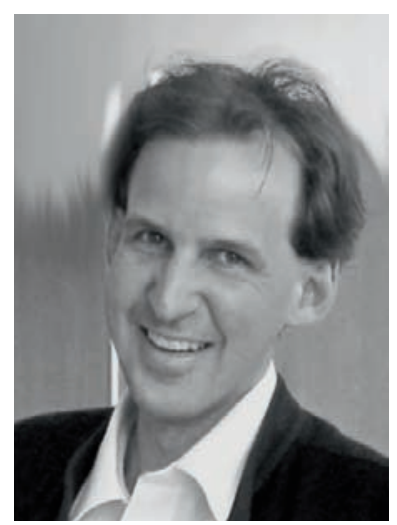

Dr. Johannes Fleckenstein,

Bern, Schweiz

\section{«Integrative Medizin»: Mainstream oder Subkultur?}

Auch in dieser Ausgabe der Schweizerischen Zeitschrift FÜr GanzheITSMEDIZIN finden Sie wieder eine breite Darstellung des komplexen Spektrums komplementärmedizinischer, integrativer Therapieoptionen.

Das National Center for Complementary and Alternative Medicine (NCCAM), eine Abteilung des US-amerikanischen Gesundheitsministeriums, beschreibt die Integrative Medizin als einen «Non-Mainstream»-Ansatz im Gesundheitswesen: Patienten werden zusätzlich zu ihrer konventionellen Therapie mit Komplementärmedizin, beispielsweise zur Symptomkontrolle, behandelt [1]. In der Einführung zu dieser Definition stolpert man über den Passus «Non-Mainstream». In der weiteren Beschäftigung mit diesem Begriff lassen sich einige Gedankengänge und Fragen ableiten:

- Die Kritik am klinischen Mainstream wird meist als Grund zur Hinwendung der Patienten zur Komplementärmedizin aufgeführt [2]. In der Musikszene wird der Begriff «Mainstream» als Abgrenzung von Independent benutzt [3]. Demzufolge wäre Komplementärmedizin im musikalischen Sinne als eine unabhängige medizinische Subkultur zu verstehen, und leider wird diese Darstellung noch zu oft verwendet. Das Zukunftsziel der Integrativen Medizin ist jedoch, die Komplementärmedizin auf der Grundlage rationaler und wissenschaftlicher Überlegungen als Selbstverständlichkeit im Kanon eines positiv bewerteten schulmedizinischen Behandlungsmainstreams mitzuverwenden. Ein Beispiel diesbezüglich wäre die aktuelle Darstellung der integrativen Sprechstunde für Gynäkologische Onkologie am Klinikum der Technischen Universität München in Deutschland [4].
- Subkulturen sind vielfältig. Die verschiedenen Disziplinen der Komplementärmedizin sind als vielfältig, als heterogen zu verstehen. Damit macht man sich zu einem gewissen Grad angreifbar, sowohl innerhalb als auch ausserhalb der eigenen Gemeinschaft. Bezogen auf die musikalische Subkultur bedeutet das: Nicht jeder aus der Independent-Szene, der «Punk» hört, sieht auch in «Electro» eine Musikrichtung. Dieses Bild lässt sich gänzlich in die Medizin übertragen. Intern sind sich Komplementärmediziner oft uneins über Wirkmechanismen, Indikationen und Nutzen der jeweiligen Therapien. Wie in jedem anderen Wissenschaftsgebiet ist die Angst zu gross, dass das eigene Verfahren, die eigene Technik, am Ende einer anderen unterlegen sein könnte. Es besteht das Risiko, den rational-wissenschaftlichen Austausch $\mathrm{zu}$ vermeiden. Es besteht die Gefahr, sich einen eigenen Elfenbeinturm zu errichten [5]. In der Vielfalt liegt jedoch auch eine reizvolle Zukunftsvision: andere Kollegen zu stärken, die Komplementärmedizin als kreatives Arbeiten zu erleben und Integrative Medizin aus der eigenen Stärke heraus authentisch zu gestalten.

Die Abgrenzung vom Mainstream der Schulmedizin ist zumeist beidseitig. In der «klassischen Schulmedizin» hat die Komplementärmedizin oft wenig musische Berechtigung. Hierfür sind verschiedene Gründe benennbar:

(1) Komplementärmedizin ist zwar aus dem Schatten der «Hinterzimmermedizin» herausgetreten, und auf eigenen Kongressen stellt man eindrucksvoll die Bandbreite der eigenen Entwicklungen dar. Ein integrativer Ansatz - die Darstellung und wissenschaftliche Auseinandersetzung auf den internationalen Jahreskongressen der schulmedizinischen Fachgesellschaften - ist hingegen noch zu selten.

(2) Die Zahl wissenschaftlicher Arbeiten ist inhomogen. Werden viele Arbeiten in komplementärmedizinischen Zeitschriften veröffentlicht, so finden sich in den jeweiligen schulmedizinischen Äquivalenten noch zu wenig Artikel.

(3) Hinzu kommt eine sogenannte "publication bias». Komplementärmedizinische Studien werden beispielsweise von etablierten Zeitschriften seltener angenommen, die Anzahl von Befürwortern ist geringer usw. Hinzu kommt der berechtigte Vorwurf, dass vor allem viele alte Studien methodologisch nicht den gegenwärtigen Ansprüchen genügen und ihrerseits einen «bias» tragen [6].

(4) Ungefähr 300000 Ärzte und Therapeuten in Europa bieten Komplementärmedizin an [7]. Alleine auf

\section{KARGER}

Fax +497614520714

Information@Karger.com www.karger.com
() 2014 S. Karger GmbH, Freiburg

1015-0684/14/0266-0324\$39.50/0

Accessible online at:

www.karger.com/szg
Dr. Johannes Fleckenstein

Abteilung für TCM/Akupunktur

Institut für Komplementärmedizin IKOM, Universität Bern

Imhoof-Pavillon, Inselspital, 3010 Bern, Schweiz

johannes.fleckenstein@ikom.unibe.ch 
der Basis dieser klinischen Routine, beispielsweise durch die Beobachtung in klinischen Registern, könnte sich das Anwendungsspektrum und Vorgehen deutlich übersichtlicher und auf einer schulmedizinisch anerkannten Basis darstellen [8]. Initiativen dieser Art erfordern jedoch Beteiligung und eine unabhängige finanzielle Unterstützung.

- Von wirtschaftlicher Seite aus betrachtet steht «Independent» für Unabhängigkeit, aber auch für eine Abkehr vom globalisierten Markt. Das trifft innerhalb der Komplementärmedizin längst nicht mehr auf jeden Kollegen zu. Medizinisch-industrielle Verwicklungen haben in der Vergangenheit zu mehr als einer fraglichen Therapieoption in der Medizin geführt [9]. Dies hat auch dem Ansehen der Komplementärmedizin geschadet [10]. Hinzu kommt, dass diejenigen komplementärmedizinischen Verfahren, die insbesondere aus dem Wissen, der Erfahrung und dem Vermögen der Therapeuten sowie der persönlichen Zuneigung zum Patienten schöpfen, aus industrieller Sicht wenig investitionswürdig sind.

Nichtsdestotrotz ist eine Finanzierung unabdingbar, um Standards in der Lehre und Ausbildung aufrechtzuerhalten und um solides wissenschaftliches Arbeiten zu ermöglichen. Die komplementärmedizinische Gemeinschaft hat ihre Möglichkeiten diesbezüglich, d.h. externe transparente Quellen - kompetitive Fördergelder, staatliche Mittel, Lobbyismus, Zusammenarbeit mit verlässlichen industriellen Partnern - sowie interne Möglichkeiten, beispielsweise Crowdfunding oder Verbundprojekte, noch nicht ausgeschöpft.

Diese Gedankengänge reflektieren unvollständig eine bisher äusserst erfolgreiche Entwicklung der Integrativen Medizin. Sie weisen aber auch auf die relevanten Themen des Heute hin, auf die wir unsere Aufmerksamkeit lenken müssen und für deren Lösung wir dringend neue Strategien benötigen. Der Weg der Integrativen Medizin kann nur dahingehen, dass er im Mainstream als wertvoller überlegter Teil wahrgenommen und genutzt wird. Man muss akzeptieren, dass es auch innerhalb der Integrativen Medizin Fehlentscheidungen geben kann und dass sich diese wie jede wissenschaftliche Richtung entsprechend anpassen und wandeln muss. Dazu gehört ebenso die Selbstreflexion der einzelnen Akteure. Ein starker Zusammenhalt wird die allgemeine schulmedizinische Resonanz auf die Integrative Medizin verbessern. Daraus entstehen neue Forschungsideen, Forschungsarbeiten und Forschungsberichte. Und letztlich ist es nicht verkehrt, sich auf einer Basis transparenten gegenseitigen Vertrauens von externen Partnern stützen und unterstützen zu lassen.

Am 23. Januar 2015 feiert das Institut für Komplementärmedizin (IKOM) der Universität Bern sein 20-jähriges Bestehen. Seit 20 Jahren arbeiten die Mitarbeiter dort gemeinsam mit verschiedensten Partnern an einer soliden Basis für eine offene, mitreissende Integrative Medizin. Ich lade Sie ein, dort zusammenzukommen, sich den selbstkritischen Fragen zu stellen sowie die gemeinsame Zukunft zu diskutieren und zu gestalten (www.kas.unibe.ch/ikom2015/).

\section{Literatur}

1 NCCAM: Complementary, Alternative, or Integrative Health: What's in a Name? National Center for Complementary and Alternative Medicine, 2014. http://nccam.nih.gov/health/whatiscam (letzter Aufruf: 10.10.2014).

2 Ernst E: The public's enthusiasm for complementary and alternative medicine amounts to a critique of mainstream medicine. Int J Clin Pract 2010;64:1472-1474.

3 Holert T, Terkessidis M: Mainstream der Minderheiten. Pop in der Kontrollgesellschaft. Berlin/Amsterdam, ID, 1996, p 190.

4 Paepke D, Klein E, Paepke S, Ettl J, Schmalfeldt B, Kiechle M: Integrative Sprechstunde für Gynäkologische Onkologie. Frauenarzt 2014;55:674676.

5 Kleiner M: Wie die Wissenschaft die Gesellschaft trägt. Forschung 2007; 3:I-VII.

6 Vannacci A, Ravaldi C, Cosci F: Publication bias in complementary and conventional medicine. Eur J Clin Pharmacol 2005;61:161-162; author reply 163.

7 Von Ammon K, Frei-Erb M, Cardini F, et al: Complementary and alternative medicine provision in Europe - first results approaching reality in an unclear field of practices. Forsch Komplementmed 2012;19(suppl 2): 37-43.

8 Feichtinger T, Fleckenstein J: Acuraid: introduction to a scientific and clinical acupuncture database: a non-profit academic project of the Department of Traditional Chinese Medicine and Acupuncture, University of Bern, Switzerland. Dtsch Z Akupunkt 2014;57:30-31.

9 Chalmers I: From optimism to disillusion about commitment to transparency in the medico-industrial complex. J R Soc Med 2006;99:337-341.

10 Lubbadeh J: Schmutzige Methoden der sanften Medizin. Süddeutsche Zeitung, 2012. www.sueddeutsche.de/wissen/homoeopathie-lobby-im-netzschmutzige-methoden-der-sanften-medizin-1.1397617. 